\title{
SPECTRAL PROCESSING FOR THE EXTRACTION OF FRACTAL PARAMETERS FROM SAR DATA
}

\author{
Gerardo Di Martino, Giorgio Franceschetti, Daniele Riccio, Ivana Zinno \\ Università di Napoli Federico II, Via Claudio 21, 80125, Napoli, Italy \\ \{gerardo.dimartino,gfrance, daniele.riccio, ivana.zinno\}@unina.it
}

\begin{abstract}
In this paper a fractal based processing for the analysis of SAR images of natural surfaces is presented. Its definition is based on a complete direct imaging model developed by the authors. The application of this innovative algorithm to SAR images makes possible to obtain a complete map of the fractal dimension of the observed scene. The retrieving is based on the estimation of the power spectrum of the SAR amplitude image. In the last section of the paper a parametric study on simulated SAR images is presented, aimed, on the one hand, to the validation of the theoretical results, and, on the other hand, to the identification of the optimal values for parameters involved in the estimation process in different situations of practical interest.
\end{abstract}

Index Terms - Fractals, Synthetic Aperture Radar, Spectral estimation

\section{INTRODUCTION}

The last generation of Synthetic Aperture Radar (SAR) sensors marked a huge increase in the resolution of microwave images of the Earth. As a matter of fact, TerraSAR-X and COSMO-SkyMed are providing SAR data with resolution of $1 \times 1 \mathrm{~m}^{2}$ in the high resolution spotlight operational mode. This significant development calls for the definition of new models in order to provide effective processing techniques for the retrieving of value added information from SAR data. However, this huge increase in the resolution implies also an increase in the number of samples of the SAR signal which can be effectively used for elaboration purposes. For processing techniques based on spectral estimation and analysis, i.e. techniques for which the number of available samples is a fundamental constraint, this can be of key importance.

In [1] the authors presented a direct model for the imaging of natural surfaces. The developed model takes into account both the interaction between the surface and the incident electromagnetic wave, through the use of appropriate fractal scattering models, and the effect of the SAR impulse response. Our analysis is based on a sound direct modeling of the observed surface and of the SAR imaging process. In particular, the observed natural surface is modeled as a fractal two-dimensional stochastic process [2], which is exhaustively described by its independent fractal parameters. In particular, the fractal dimension $D$ of an observed surface is strictly related to the roughness and geophysical characteristics of the surface and its knowledge can be of key importance for a wide range of applications, including the prevention and monitoring of environmental disasters, land classification, rural and urban planning and so on. The estimation of this parameter from a SAR image can be accomplished, in an appropriate range of frequencies, through a linear regression on the $\log -\log$ spectrum of a range cut of the SAR image, as shown in [1].

In this paper the inversion of this imaging model is detailed, with particular focus on the spectral estimation step. In fact, spectral estimation in the fractal, power law spectrum case is not a trivial task, because of the presence of leakage and high variance effects which strongly affect classical estimators [3]. In this paper we present the rationale of the Capon estimator [3], [4] and we discuss the role of the parameters involved in this spectral estimation method.

The paper is organized as follows. In Section 2 we introduce the theoretical aspects of the proposed imaging model. In Section 3 the methodological framework is discussed: in particular, the implementation of the Capon estimator and the overall scheme of our processing steps are presented. In Section 4 a parametric study, aimed to the identification of the optimal values for the involved parameters in various situations of practical interest, is performed on simulated data. Finally, in Section 5 significant conclusions are highlighted.

\section{THEORETICAL FRAMEWORK}

It is widely recognized that fractal models represent the best way to describe the irregularity of natural scenes [2], [5]. Among this kind of models, we choose the regular stochastic fractional Brownian motion $(\mathrm{fBm})$ process that completely describes natural surfaces by means of two independent parameters: the Hurst coefficient, $H$ (which is related to the fractal dimension by the simple relation $D=3$ $H$ ) and the standard deviation of surface increments at 
unitary distance, $s\left[\mathrm{~m}^{1-H}\right]$. The process $z(x, y)$ is an $\mathrm{fBm}$ if, for every $x, y, x^{\prime}, y^{\prime}$, it satisfies the following relationship:

$$
\begin{gathered}
\operatorname{Pr}\left\{z(x, y)-z\left(x^{\prime}, y^{\prime}\right)<\bar{\zeta}\right\}=\frac{1}{\sqrt{2 \pi} s \tau^{H}} \int_{-\infty}^{\bar{\zeta}} \exp \left(-\frac{\zeta^{2}}{2 s^{2} \tau^{2 H}}\right) d \zeta \\
\tau=\sqrt{\left(x-x^{\prime}\right)^{2}+\left(y-y^{\prime}\right)^{2}}
\end{gathered}
$$

The power density spectrum of the isotropic two dimensional $\mathrm{fBm}$ process exhibits an appropriate power-law behavior [5]:

$$
S(k)=S_{0} k^{-\alpha},
$$

wherein $S_{0}$ and $\alpha$ are functions of the fractal parameters [5].

Besides the surface model, in order to retrieve the fractal dimension of a natural scene starting from its SAR image we need a direct model relating the surface to its final amplitude image. In [1] the authors presented a complete imaging model that links the fractal parameters of the surface to the stochastic parameter of the image, via reliable electromagnetic and radar models. In the hypothesis of small slope regime for the observed surface, the image amplitude comes out to be a linear function of the partial derivative of the surface evaluated along the range direction.

The expressions of the autocorrelation functions of the SAR image and of the Power Spectral Densities (PSDs) of two cuts of the image in the range and azimuth directions respectively, have been evaluated by the authors in [1]. The PSDs of the azimuth and of the range cut of the SAR image show very different behaviors, thus highlighting an intrinsic asymmetry in the structure of SAR data, which is also intuitively referable to the particular acquisition geometry of a side looking mono-static radar. In particular, the spectrum of the image range cut, in an appropriate range of sufficiently low spatial frequencies, presents a power law behavior - thus showing in a log - log plane a linear behavior with a slope related to the Hurst coefficient $H$ of the observed surface. In fact, the expression of the PSD of the range cut of a SAR image, for adequately low wavenumbers, turns out to be proportional to the PSD of the range cut derivative process, which can be written as [1]:

$$
S_{p}\left(k_{y}\right)=s^{2} \Gamma(1+2 H) \operatorname{sen}(\pi H) \frac{1}{\left|k_{y}\right|^{2 H-1}},
$$

where $k_{y}$ is the wavenumber of the range cut of the image and $\Gamma$ is the Euler Gamma function.

From Eq. (3) it can be stated that the spectrum of interest presents a power law behavior, whose exponent is simply related to the Hurst coefficient of the observed surface.

\section{METHODOLOGICAL SETUP}

Starting from Eq. (3), it is possible to implement linear regression techniques on the spectrum of range cuts of the image in a $\log ($ wavenumber $)-\log (\mid$ spectrum $\mid)$ plane, in order to retrieve the fractal dimension of the imaged surface.

However, in order to apply the inversion algorithm on actual SAR amplitude images, we must deal with the estimation of the power density spectrum. The analysis of actual SAR images of natural surfaces calls for spectral estimators which are able to effectively work with a small number of samples: in fact, the fractal dimension is a local property of the surface and can exhibit significant variations depending on both the position and the scales taken into account. Hence, the estimation of the fractal parameters of a physical fractal surface from the relevant SAR image requires the use of a sliding window of small dimension in order to obtain an adequate resolution for the map of the retrieved values.

In the following sub-section we summarize the rationale of the spectral estimation method used throughout the paper.

\subsection{Capon spectral estimator}

The spectrum estimation problem is not a trivial one: as a matter of fact, power law spectra introduce unique difficulties in spectral estimation as they greatly suffer leakage effects and high variance problems, which can give rise to deep modifications of the original spectral slope. In order to reduce the variance of the spectral estimates the use of large data sets allowing the evaluation of average spectra would be desirable. In our case the limited dimension of the data set, due to the use of a relatively small sliding window, requires to resort to a spectral estimator able to reduce as much as possible both leakage and variance of the estimate also in presence of a small number of samples [3]. Hence, for our application the use of classical spectral estimators, such as the periodogram obtained using Fourier-based techniques, does not represent a good solution. Conversely, the Capon estimator [3], [4] strongly reduces the abovementioned negative effects and is particularly well suited when facing short data records.

The Capon estimator essentially performs a filtering which is customized for each frequency of interest and which is aimed to minimize the total power output, with the constraint that the gain at the frequency of interest is unity. In particular, in the power law case the sidelobes of the filter are adjusted in order to avoid leakage from low frequency components. In order to evaluate the power spectrum, the Capon method requires the evaluation of an estimate of the autocorrelation matrix. In this paper we use the covariance method, which provides an unbiased and consistent estimator [4]. According to this method the elements of the autocorrelation matrix relevant to a one-dimensional range cut of our SAR image, $I$, of which $N$ samples are available, can be estimated as: 


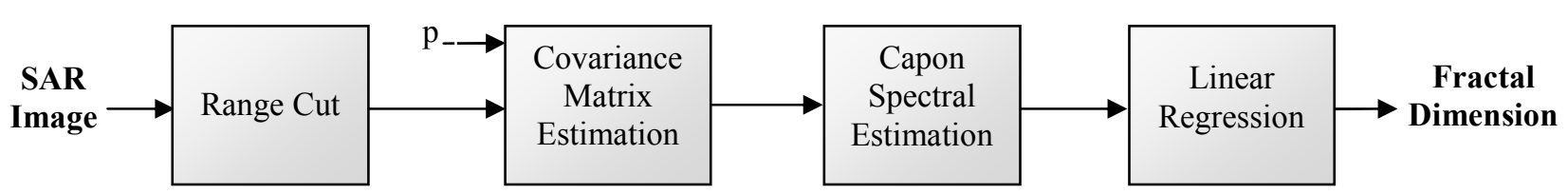

Fig. 1. Block diagram of the spectral estimation and of the extraction of the fractal dimension.

$$
\begin{aligned}
{\left[\hat{R}_{I}\right]_{i j}=\frac{1}{2(N-p)}[} & {\left[\sum_{n=p}^{N-1} I[n-i] I[n-j]\right.} \\
& \left.+\sum_{n=0}^{N-1-p} I[n+i] I[n-j]\right] .
\end{aligned}
$$

The Capon estimate of the spectrum of interest can be then computed as:

$$
\hat{S}_{p}\left(k_{y}\right)=\frac{p \Delta y}{\mathrm{e}^{*} \hat{R}_{I}^{-1} \mathrm{e}^{\prime}}
$$

wherein $p$ is the dimension of the autocorrelation matrix, $\Delta y$ is the ground range resolution of the SAR image, * stands for conjugate transpose and

$$
\mathbf{e}=\left[\begin{array}{lllll}
1 & e^{j 2 \pi k_{y} \Delta y} & e^{j 4 \pi k_{y} \Delta y} & \ldots & e^{j 2 \pi(p-1) k_{y} \Delta y}
\end{array}\right]^{T} .
$$

The bias of the Capon estimator is independent of $N$, but it is dependent on $p$. An increase in $p$ determines a decrease in the bias at the expense of an increased variance. Indeed, note that $p \Delta y$ is the longest lag for which the autocorrelation is estimated and, obviously, the range of $p$ is constrained by $N$. For this estimator the region of high accuracy for the estimated spectra is $1 /(2 p \Delta y)<k_{y}<$ $1 /(2 \Delta y)$. Different values of $p$ will be tested in the experimental setup in order to analyze the behavior of the obtained spectral estimated as a function of this parameter.

However, also the aliasing, due to the fact that the imaged surface will generally have frequency components at spatial frequencies above the Nyquist frequency, $f_{s}=$ $1 /(2 \Delta y)$ should be considered. Hence, according to the literature on the subject [3], we discard the values of the spectral estimate computed for normalized wavenumbers greater than $f_{s} / 2$.

\subsection{Algorithm implementation}

The implemented algorithm extracts the local fractal dimension of the imaged surface working on patches of the SAR image using a moving window spanning the whole image. The dimension of this elaboration window can be set by the user according to its specific needs, resulting from a trade-off between accuracy and resolution of the output fractal dimension map.
In particular, the algorithm performs spectral estimation selecting in each window range cuts that are sufficiently spaced from each other to be considered uncorrelated. Then the spectra of these cuts (whose number can be again chosen by the user, resulting from a trade-off between accuracy and computation time) are evaluated and averaged using the Capon spectral estimator described in the previous subsection. Finally, a linear regression is performed on this average PSD.

As a result, we obtain a map of the fractal dimension of the observed scene: the resolution of this map depends on both the resolution of the input image (the higher the resolution of the image, the better the resolution of the map) and the dimension of the estimation window. A block diagram of the proposed algorithm is reported in Fig. 1.

Furthermore, we cannot set completely aside the speckle phenomenon, which is responsible for the wellknown salt and pepper effect on SAR amplitude images. As a matter of fact, radar single-look images hold small scale spatial properties dominated by the speckle effect, hence in the wavenumber domain the high frequency range of the image spectrum is degraded. However, our algorithm performs the linear regression in a range of spatial frequencies in which the spectrum is not significantly affected by this phenomenon.

In the next section, we show a parametric study aimed at the identification of the best values for the above introduced parameter $p$ in case of data sets of different dimensions and different values of the fractal dimension. The algorithm is applied on simulated SAR images of canonical surfaces presenting user-defined fractal parameters.

\section{EXPERIMENTAL SETUP}

In order to validate and to test the theoretical and methodological frameworks described in the previous sections, a large set of applications relevant to canonical fractal cases is presented in this section.

The proposed SAR image post-processing is applied to simulated SAR images of canonical fractal surfaces presenting controlled fractal parameters. Thus, the estimate of the Hurst coefficient performed with our algorithm can be compared with the actual one.

First, an $\mathrm{fBm}$ surface of known fractal parameters is synthesized using a Weierstrass-Mandebrot function [5] (Fig. 2). This fractal canonical surface is used as digital elevation model (DEM) for a SAR image simulator, the SARAS [6], which provides the simulated SAR image of the fractal surface given as input (Fig. 3). 


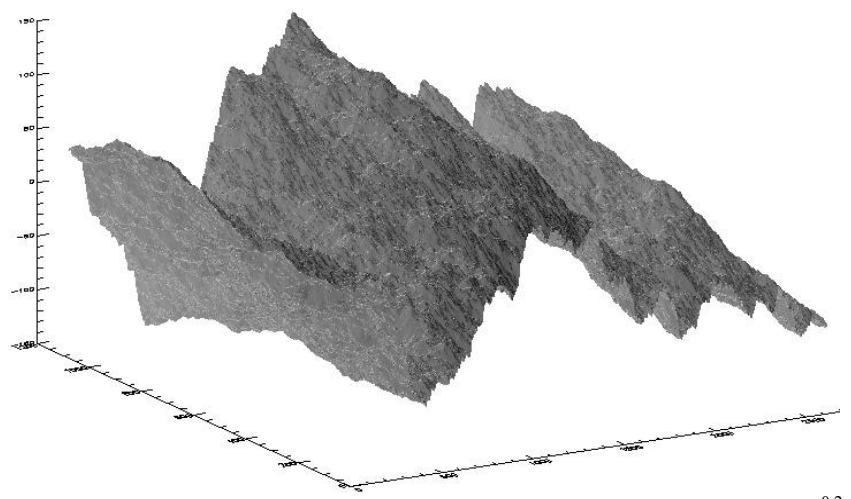

Fig. 2. Canonical fBm Surface of fractal parameters $H=0.8, s=0.1 \mathrm{~m}^{0.2}$ synthesized using a Weierstrass-Mandebrot function.

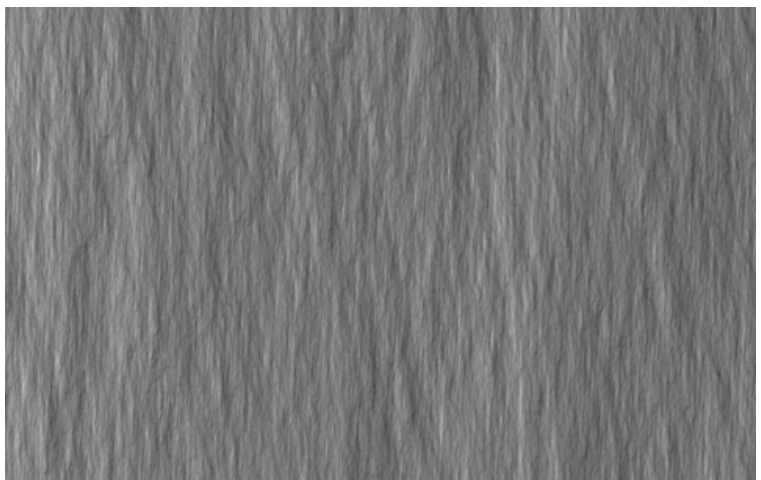

Fig. 3. Simulated SAR Image of the Fractal Surface given in Fig. 2

In order to prove the goodness of the retrieving algorithm, the SAR image processing described in Fig. 1 is applied to a set of SAR images with different fractal parameters. Furthermore, in the aforementioned cases, in order to test the performances of the estimation, we present the results of a parametric study, using different values for the length of the Capon filtering $p$ and two different linear regression techniques.

A key point of the spectral estimation is the dimension of the data segment on which the estimation is performed. We first apply our SAR image processing on images of 1000x1000 pixels: depending on the chosen Capon filter length, a different number of the original available samples is used, so this case let us understand which is the optimal value for the parameter $p$.

Then, with regard to the analysis of actual SAR images, we have to deal with very small data segments: we apply the algorithm using windows of 50x50 pixels; this procedure is described in Section 4.2.

\subsection{Application to $1000 \times 1000$ pixels simulated SAR images}

The results presented in Tab. I-III are relevant to simulated ENVISAT SAR images of $1000 \times 1000$ pixels of canonical surfaces having the same fractal parameters at all scales. We have studied the case of fractal surfaces with the same value of $s=0.1\left[\mathrm{~m}^{1-H}\right]$ and different values of the Hurst coefficient: $H=0.7, H=0.8, H=0.9$. For all the three cases the results relevant to the application of the Capon spectral estimation in the case of:

- no filtering (Tab. I)

- length of filtering $=0.3 * N$ (Tab. II)

- length of filtering $=0.1 * N$ (Tab. III)

are presented. In all cases an anti-aliasing filtering is performed on the estimated spectra, that consists in discarding the frequencies greater than $f_{s} / 2$.

Furthermore, we have applied two different linear regression techniques:

- linfit: minimization of the chi-square error statistic

- ladfit: "robust" least absolute deviation method

in order to compare the performances. Only for the sake of brevity, we present only the results relevant to the linfit case, which is the one that perform the better estimation in all the cases. Besides, the linfit procedure allows the evaluation of the unreduced chi-square goodness-of-fit statistic, whose values are also reported in Tab. I-III. The obtained estimated samples of the spectra are shown in Fig. 4 compared with the theoretical ones.

TABLE I

NO FILTERING CASE APPLIED TO A 1000X1000 PIXELS SAR IMAGE

\begin{tabular}{lcccc}
\hline \hline & $S\left[\mathrm{~m}^{1-\mathrm{H}}\right]$ & $H$ & estimated $H$ & chi-square \\
\hline Fig. 4 (A) & 0.1 & 0.7 & 0.73 & 3.87 \\
Fig. 4 (B) & 0.1 & 0.8 & 0.83 & 2.60 \\
Fig. 4 (C) & 0.1 & 0.9 & 0.89 & 1.74 \\
\hline \hline
\end{tabular}

TABLE II

CAPON FILTERING OF LENGTH $0.3 * N($ LENGTH $=300$ SAMPLES $)$

\begin{tabular}{lcccc}
\hline \hline & $s\left[\mathrm{~m}^{1-\mathrm{H}}\right]$ & $H$ & estimated $H$ & chi-square \\
\hline Fig. 4 (D) & 0.1 & 0.7 & 0.73 & 2.5 \\
Fig. 4 (E) & 0.1 & 0.8 & 0.83 & 1.71 \\
Fig. 4 (F) & 0.1 & 0.9 & 0.90 & 1.32 \\
\hline \hline
\end{tabular}

TABLE III

CAPON Filtering OF LeNGTH $0.1 * N($ Length $=100$ SAMPLeS $)$

\begin{tabular}{ccccc}
\hline \hline & $\boldsymbol{s}\left[\mathrm{m}^{1-\mathrm{H}}\right]$ & $\boldsymbol{H}$ & estimated $\boldsymbol{H}$ & chi-square \\
\hline Fig. 4 (G) & 0.1 & 0.7 & 0.75 & 1.22 \\
Fig. 4 (H) & 0.1 & 0.8 & 0.85 & 0.73 \\
Fig. 4 (I) & 0.1 & 0.9 & 0.92 & 0.69 \\
\hline \hline
\end{tabular}



(A) 


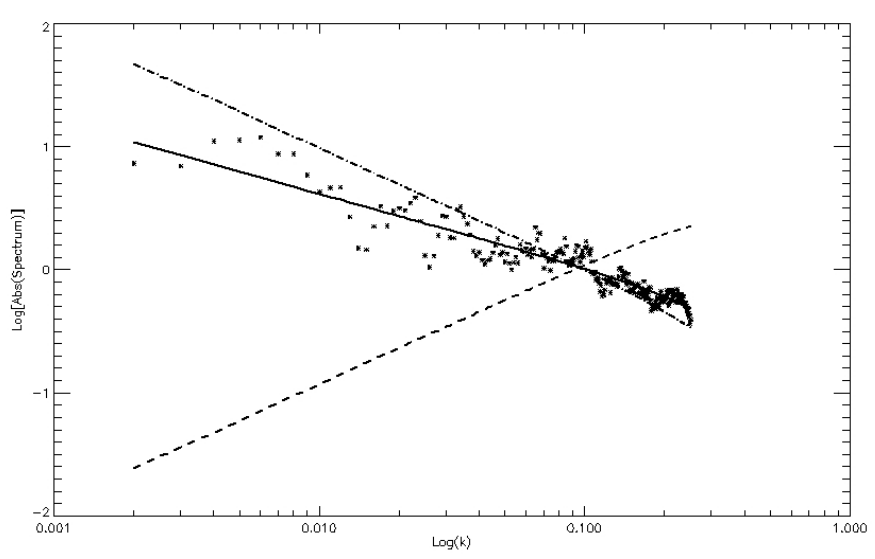

(B)

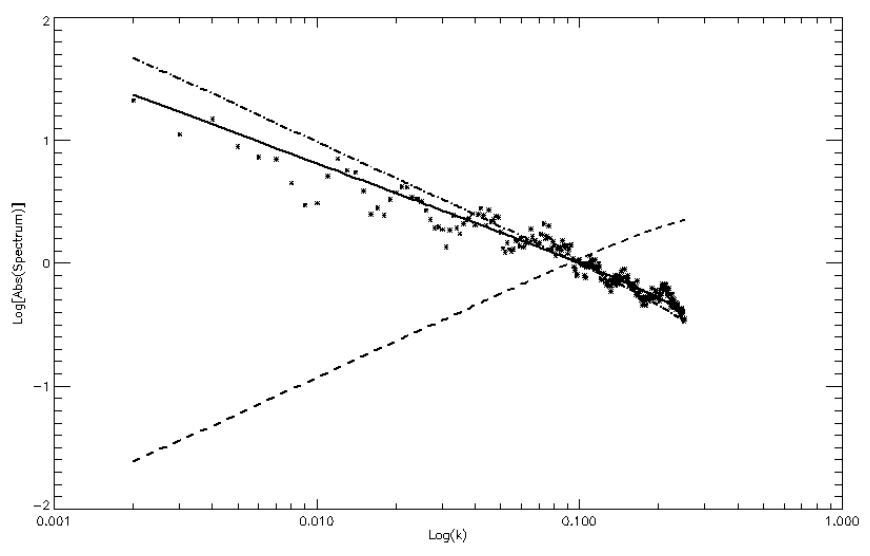

(C)

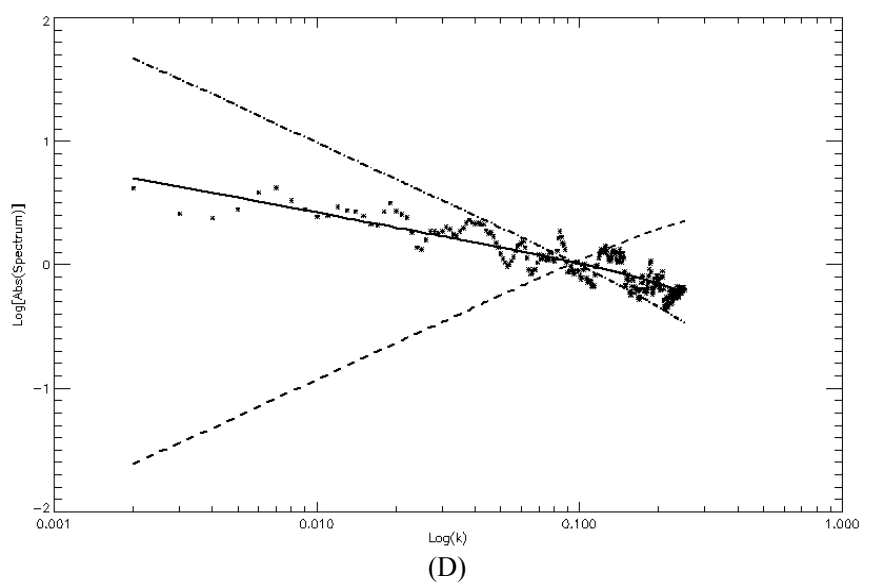

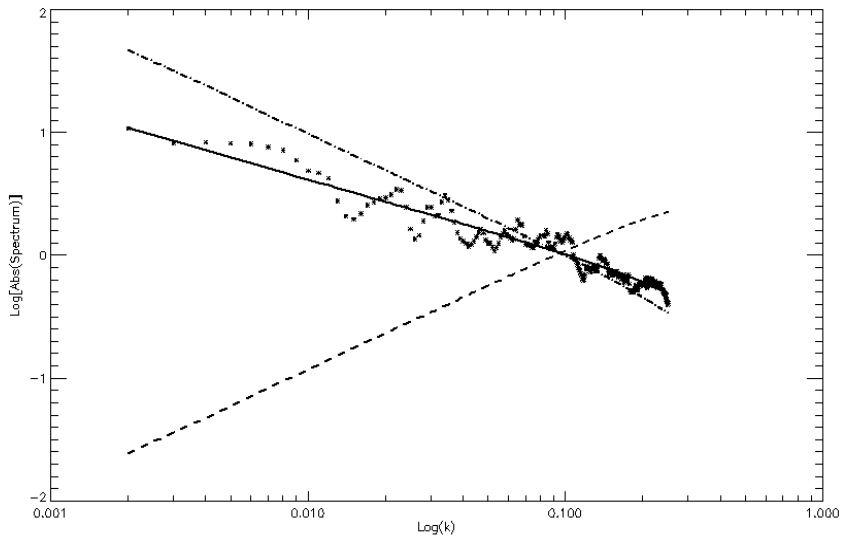

(E)
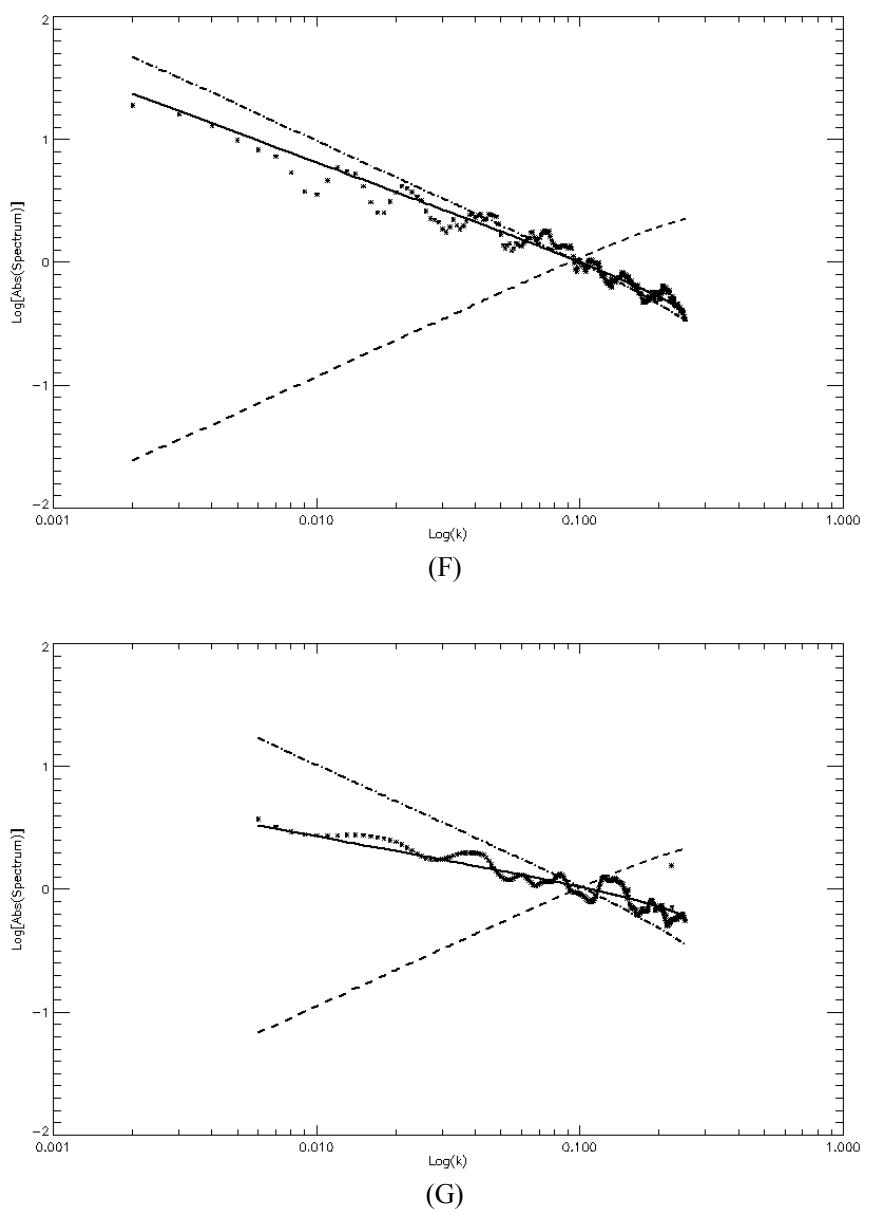


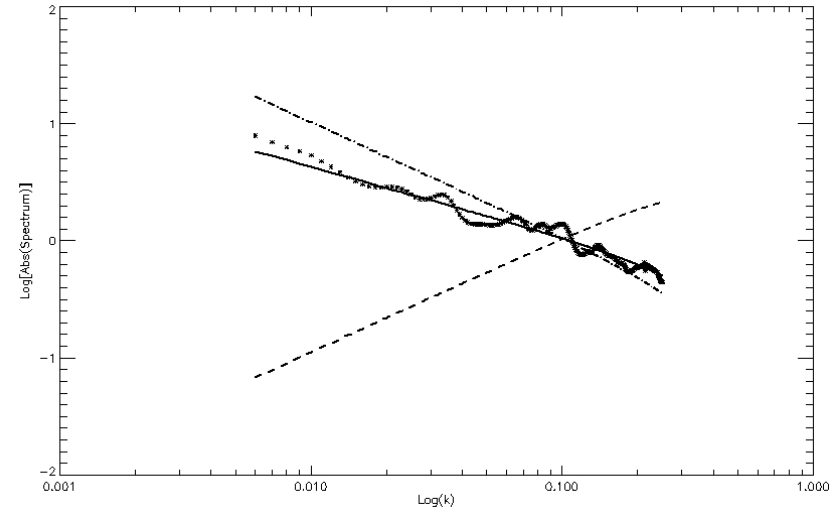

(H)

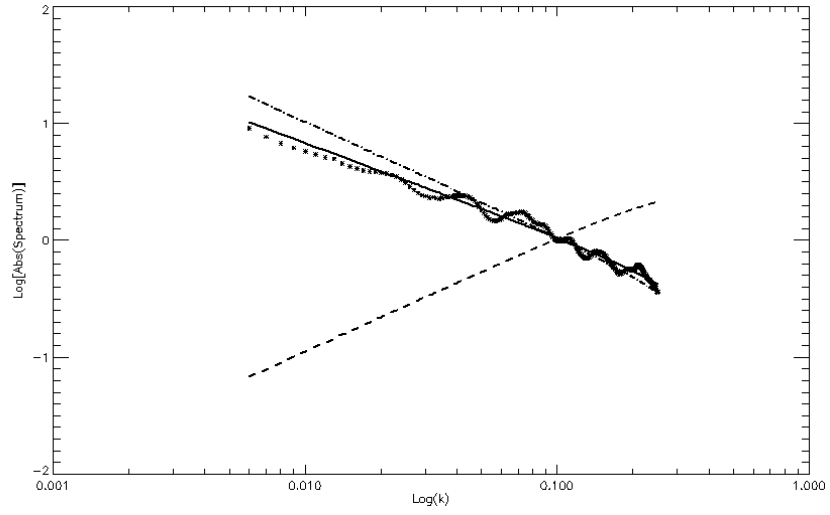

(I)

Fig. 4. Estimated (points) and theoretical (continuous line) PSDs of a range cut of the 1000x1000 pixels of a surface, whose fractal parameters are defined in Tables I-III The dashed lines delimit the range of slopes allowed for a fractal.

From the results in Tab. I-III, it can be deduced that the best estimation is performed when the Capon estimator is applied with a filter length equal to $0.3^{*} N$. Besides, it is worth stressing that, even if the chi-square is reduced when the filter length decreases, the bias increases and so the estimate worsen.

\subsection{Application to simulated SAR images cuts-out of $50 \times 50$ pixels}

In order to apply our processing to actual SAR images, we have to deal with the problem of analyzing very small areas in which the fractal parameters can be considered constant. Hence, in this subsection, the algorithm is applied to cutsout of 50x50 pixels of the SAR images considered in the previous sub-section. Obviously, in this case, there are very few samples available to perform the spectrum estimation, so the results of the retrieving technique are not so good as in the previous case. In Tab. IV-VI the results relevant to the processing performed without the Capon filtering, with a Capon filtering with $p=0.3 * N$ (filter length $=15$ samples) and with $p=0.1 * N$ (filter length $=5$ samples) are shown. We stress that the estimated values in Tab. IV-VI are averaged on several elaboration windows taken from different areas of each simulated image.

TABLE IV

No FiLTERING CASE APPLIED TO A 50X50 PIXELS SAR IMAGE CUT-OUT

\begin{tabular}{cccc}
\hline \hline$s\left[\mathrm{~m}^{1-\mathrm{H}}\right]$ & $H$ & estimated $H$ & chi-square \\
\hline 0.1 & 0.7 & 0.74 & 0.17 \\
0.1 & 0.8 & 0.85 & 0.14 \\
0.1 & 0.9 & 0.86 & 0.18 \\
\hline \hline
\end{tabular}

TABLE V

CAPON FILTERING OF LENGTH $0.3 * N($ LENGTH= 15 SAMPLES $)$

\begin{tabular}{lcccc}
\hline \hline & $\boldsymbol{s}\left[\mathrm{m}^{1-\mathrm{H}}\right]$ & $\boldsymbol{H}$ & estimated $\boldsymbol{H}$ & chi-square \\
\hline Fig. 3 (A) & 0.1 & 0.7 & 0.74 & 0.06 \\
Fig. 3 (B) & 0.1 & 0.8 & 0.84 & 0.05 \\
Fig. 3 (C) & 0.1 & 0.9 & 0.92 & 0.08 \\
\hline \hline
\end{tabular}

TABLE VI

CAPON FILTERING OF LENGTH $0.1 * N($ LENGTH $=5$ SAMPLES $)$

\begin{tabular}{cccc}
\hline \hline $\boldsymbol{s}\left[\mathrm{m}^{1-\mathrm{H}}\right]$ & $\boldsymbol{H}$ & estimated $\boldsymbol{H}$ & chi-square \\
\hline 0.1 & 0.7 & 0.81 & 0.002 \\
0.1 & 0.8 & 0.87 & 0.002 \\
0.1 & 0.9 & 0.93 & 0.001 \\
\hline \hline
\end{tabular}

For the sake of brevity, hereafter only the plots of the range cut PSDs relevant to the case of the best filtering, i.e. $p=0.3^{*} N$, are presented. It is worth stressing that, due to the Capon filtering and then to the antialiasing filtering performed on an already short data segment, the numer of samples on which the spectrum estimation is performed is significantly reduced. In Fig. 5, the PSDs relevant to the results in Tab. V are shown: in this case the number of samples used for the estimation is equal to 11 .

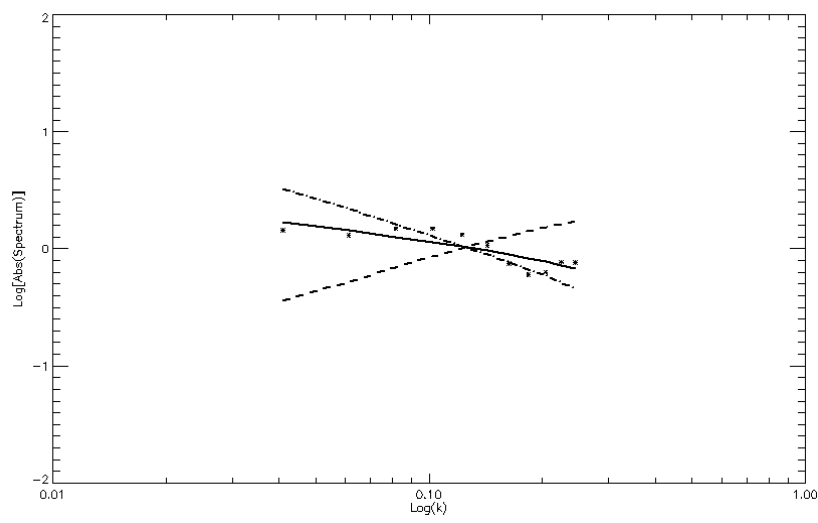

(A) 


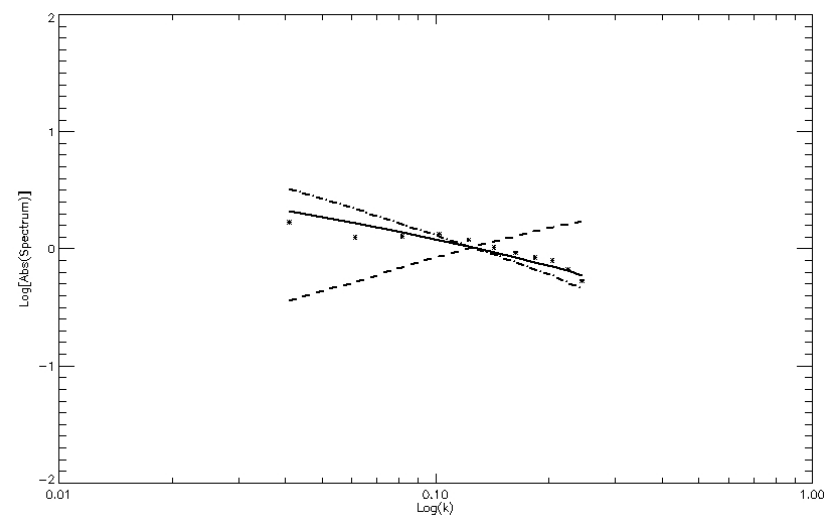

(B)

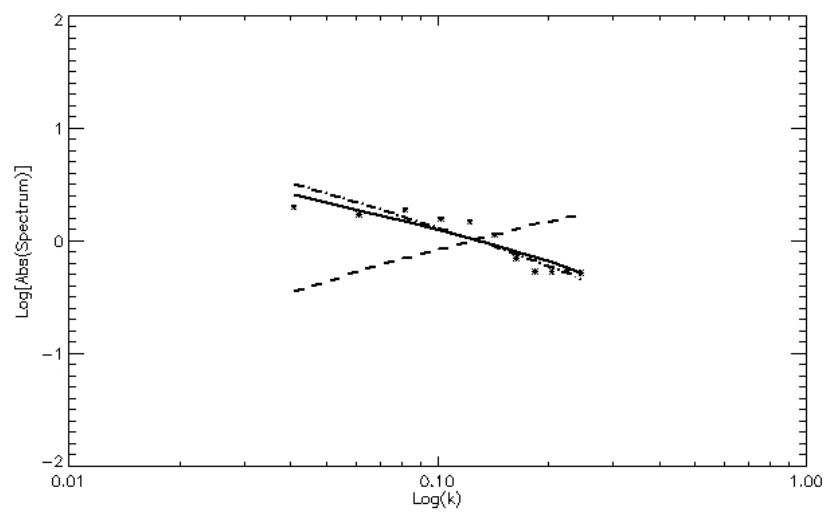

(C)

Fig. 5. Estimated (points) an theoretical (continue lines) PSDs of a range cut of a 50x50 pixels cut-out of a SAR image of a surface of fractal parameters given in Tab.V. The dashed lines delimit the range of slopes of a fractal.

Obviously, due to the small number of samples used for the estimation, the results relevant to small data segments (Tab. IV-VI) are generally worse than the results of the previous section (Tab. I-III). Nevertheless, also for small data segments, the best filter length is equal to $0.3 * \mathrm{~N}$. Indeed, the trend of the results in Tab. IV-VI is the same of that in Tab. I-III: the chi-square decreases if the filter length increase, but the bias increases too, so, for the cases at hand, it is not convenient to use a filter length smaller than $0.3^{*} N$.

\section{CONCLUSIONS}

In this paper an innovative fractal based processing for the analysis of SAR images of natural surfaces has been presented. It is based on the inversion of a complete direct imaging model developed by the authors. The application of this innovative algorithm to SAR images makes possible to obtain a complete map of the fractal dimension of the observed scene. The fractal dimension retrieving is based on the estimation of the power density spectrum of range cuts of the SAR amplitude image. In order to validate the theoretical results and to identify the optimal values for the parameters involved in the estimation process, a parametric study on simulated SAR images has been presented for different situations of practical interest. Significant results, along with relevant considerations, have been presented and discussed in details.

\section{ACKNOWLEDGEMENTS}

This work has been supported by the Agenzia Spaziale Italiana (ASI) within the project "COSMO/SkyMed buildings features extraction from single SAR images: application to COSMO/SkyMed high resolution SAR images".

\section{REFERENCES}

[1] G. Di Martino, A. Iodice, D. Riccio, G. Ruello, I. Zinno, "The effects of acquisition geometry on SAR images of natural scenes", Proceedings of the Radar Conference 2009, Rome, Sept. 30-Oct. 2 2009, pp. $541-544$.

[2] B.B. Mandelbrot, The Fractal Geometry of Nature. New York: Freeman, 1983.

[3] T. Austin, A. W. England, G. H. Wakefield, "Special problems in the estimation of power-law spectra as applied to topographical modeling", IEEE Trans. Geosci. Remote Sens., vol. 32, no. 4, pp. 928-939, July 1994.

[4] S. M. Kay, Modern Spectral Estimation. Englewood Cliffs, NJ: Prentice- Hall, 1988.

[5] G. Franceschetti, D. Riccio, Scattering, Natural Surfaces and Fractals. Academic Press, Burlington (MA), USA, 2007.

[6] G. Franceschetti, M. Migliaccio, D. Riccio, G. Schirinzi, SARAS: a SAR Raw Signal Simulator, IEEE Trans. Geosci. Remote Sens., vol. 30, no. 1, pp. 110-123, Jan. 1992. 